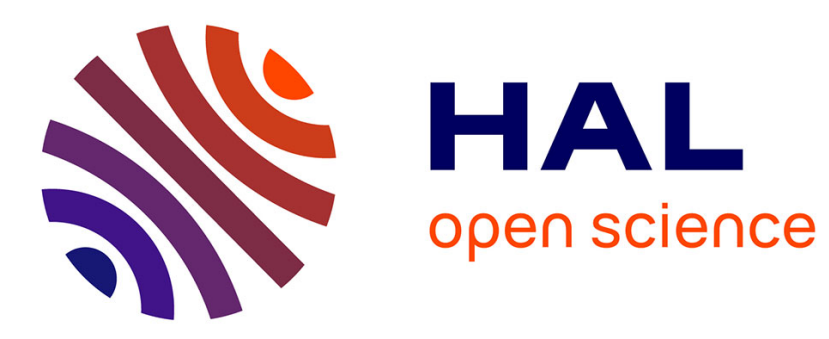

\title{
Explicitly correlated coupled cluster methods with pair-specific geminals
}

Hans-Joachim Werner, Gerald Knizia, Frederick R Manby

\section{To cite this version:}

Hans-Joachim Werner, Gerald Knizia, Frederick R Manby. Explicitly correlated coupled cluster methods with pair-specific geminals. Molecular Physics, 2010, pp.1. 10.1080/00268976.2010.526641 . hal00638527

\section{HAL Id: hal-00638527 \\ https://hal.science/hal-00638527}

Submitted on 5 Nov 2011

HAL is a multi-disciplinary open access archive for the deposit and dissemination of scientific research documents, whether they are published or not. The documents may come from teaching and research institutions in France or abroad, or from public or private research centers.
L'archive ouverte pluridisciplinaire HAL, est destinée au dépôt et à la diffusion de documents scientifiques de niveau recherche, publiés ou non, émanant des établissements d'enseignement et de recherche français ou étrangers, des laboratoires publics ou privés. 


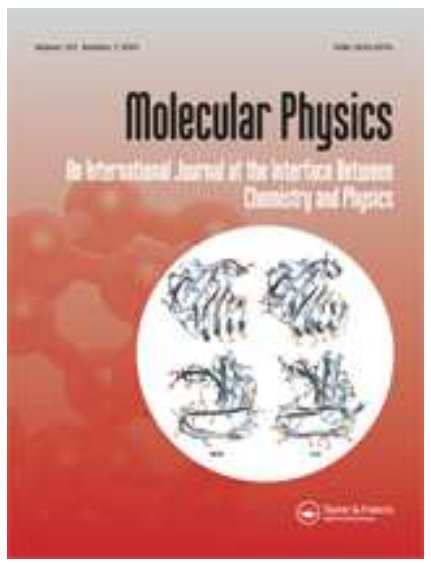

\section{Explicitly correlated coupled cluster methods with pair- specific geminals}

\begin{tabular}{|r|l|}
\hline Journal: & Molecular Physics \\
\hline Manuscript ID: & TMPH-2010-0338.R1 \\
\hline Manuscript Type: & Full Paper \\
\hline Date Submitted by the & $17-$ Sep-2010 \\
\hline Complete List of Authors: & $\begin{array}{l}\text { Werner, Hans-Joachim; Institute for Theoretical Chemistry } \\
\text { Knizia, Gerald; University of Stuttgart, Institute for Theoretical } \\
\text { Chemistry } \\
\text { Manby, Frederick; University of Bristol, Centre for Computational } \\
\text { Chemistry, School of Chemistry }\end{array}$ \\
\hline Keywords: & $\begin{array}{l}\text { explicit correlation, coupled-cluster, core-valence correlation, F12- } \\
\text { methods }\end{array}$ \\
\hline $\begin{array}{l}\text { Note: The following files were submitted by the author for peer review, but cannot be converted } \\
\text { to PDF. You must view these files (e.g. movies) online. }\end{array}$ \\
\hline mgem.tex
\end{tabular}

\section{SCHOLARONE ${ }^{m}$ \\ Manuscripts}




\section{Introduction}

During the last few years efficient and robust explicitly correlated second-order Møller-Plesset (MP2-F12) and coupled-cluster [CCSD(T)-F12] methods have been developed, and it has been demonstrated in numerous benchmarks and applications that these lead to dramatically reduced basis set truncation errors. Typically, CCSD(T)-F12 with triple- $\zeta$ basis sets yields results that show at least the accuracy of conventional $\operatorname{CCSD}(\mathrm{T})$ calculations with quintuple- $\zeta$ basis sets. Even with double- $\zeta$ basis sets the intrinsic accuracy of $\operatorname{CCSD}(\mathrm{T})$ is often reached. Details and references to earlier work can be found in recent reviews[1-3].

A common feature of most CCSD(T)-F12 methods is that the amplitudes of the Slater-type geminal are determined from the wave function cusp conditions [4, 5], as proposed by Ten-no[6, 7]. Thus, no extra equations have to be solved to determine these amplitudes. A remaining parameter in the calculations is the exponent $\gamma$ of the Slater function, $F_{12}=-\frac{1}{\gamma} e^{-\gamma r_{12}}$, which determines the size of the correlation hole modeled by the geminal. If the geminal amplitudes are fully optimized, the dependence of the energy on this parameter is rather weak; but it is more pronounced if the fixed amplitude approximation is used. For calculations in which just the valence orbitals are correlated this does not cause significant errors, and an average value of $\gamma=1.0 a_{0}^{-1}$ has been found to work well for a large range of molecules and applications[8-11]. However, if inner shells are also correlated, it is not possible to define an exponent that is appropriate for all orbital pairs. For example, in calculations involving transition metals or other post- $d$ elements, it is important to correlate the $d$-shell. Since the $d$-orbitals are much more compact

*Corresponding author. Email: werner@theochem.uni-stuttgart.de 


\section{Theory}

The MP2-F12 and CCSD(T)-F12x theory and the working equations as used in our programs have been presented in earlier work $[3,8,21,26,27]$, and the details will not be repeated here. We will therefore just outline the ansatz, and explain how the method is extended to pair-specific geminals. In the following, occupied orbitals will be denoted by indices $i, j, k, l, m, n, o, p$; virtual orbitals in the orbital basis set (OBS) by $a, b$; any orbitals in the OBS by $r, s$; and complementary auxiliary orbitals (CABS) by $x, y$. The occupied orbital indices $i \ldots p$ exclude frozen core orbitals, unless indicated otherwise. The indices $\alpha, \beta$ run over the complete virtual space, which contains the virtual $(a, b)$ and CABS $(x, y)$ orbitals as subspaces. Summation over repeated dummy indices is always implied. For simplicity, we will first consider the closed-shell case, using a spin-free formulation. We will assume that canonical Hartree-Fock orbitals are used (i.e., that the Fock matrix is diagonal in the OBS). The extension to an orbital invariant formulation[26] and open-shell[8] cases is discussed at the end of this section.

The new wave function ansatz with pair specific geminals is best shown for the MP2-F12 method, which is also the first step of a CCSD(T)-F12x calculation. The MP2-F12 first-order wavefunction is defined as [28]

$$
\begin{aligned}
\Psi^{(1)} & =\frac{1}{2}\left[\Phi_{i j}^{a b} T_{a b}^{i j}+\Phi_{i j}^{\alpha \beta}\left\langle\alpha \beta\left|\hat{Q}_{12} F_{12}^{(i j)}\right| k l\right\rangle T_{k l}^{i j}\right], \\
\Phi_{i j}^{\alpha \beta} & =\hat{E}_{i}^{\alpha} \hat{E}_{j}^{\beta} \Phi_{\mathrm{HF}} .
\end{aligned}
$$


Here $\hat{E}_{i}^{\alpha}=\eta_{\alpha}^{\dagger} \eta_{i}+\bar{\eta}_{\alpha}^{\dagger} \bar{\eta}_{i}$ are spin-summed one-electron excitation operators, and $F_{12}^{(i j)}$ is the $r_{12}$-dependent correlation factor,

$$
F_{12}^{(i j)}=-\frac{1}{\gamma} e^{-\gamma^{(i j)} r_{12}}
$$

with $\gamma^{(i j)}$ a length-scale parameter. In contrast to previous F12 treatments, we assume here that this parameter can be pair dependent, as indicated by the superscript $(i j)$. As will be discussed later, we will only use two or three different values in practice, but for the sake of simplicity and generality, we make no such restrictions in the theory.

The projector $\hat{Q}_{12}$ keeps the explicitly correlated terms strongly orthogonal to the Hartree-Fock (HF) reference function and orthogonal to the conventional singly and doubly excited configurations $\Phi_{i}^{a}$ and $\Phi_{i j}^{a b}$

$$
\hat{Q}_{12}=\left(1-\hat{o}_{1}\right)\left(1-\hat{o}_{2}\right)-\hat{v}_{1} \hat{v}_{2}
$$

where the operators $\hat{o}=\sum_{i}^{\text {occ }}|i\rangle\langle i|\equiv| i\rangle\left\langle i\left|, \hat{v}=\sum_{a}^{\text {virt }}\right| a\right\rangle\langle a|\equiv| a\rangle\langle a|$ project onto the occupied orbital space (including frozen core orbitals) and virtual orbital space, respectively. The subscripts of $\hat{o}$ and $\hat{v}$ denote the electron coordinate on which they act. In order to avoid many-electron integrals, resolution of the identity (RI) approximations are used. If the RI is spanned by the union of the OBS and an auxiliary basis set (CABS approach[29]) the projector takes the form

$$
\hat{Q}_{12}=1-|r s\rangle\langle r s|-| i x\rangle\langle i x|-| x i\rangle\langle x i|,
$$

where $i$ includes frozen core orbitals. The amplitudes $T_{k l}^{i j}$ of the explicitly correlated terms are determined by the wave function cusp conditions [4, 5], as proposed by Ten-no[6, 7$]$

$$
T_{k l}^{i j} \stackrel{\mathrm{FIX}}{=} \frac{3}{8} \delta_{i k} \delta_{j l}+\frac{1}{8} \delta_{j k} \delta_{i l}
$$

This is denoted either fixed amplitude (FIX) or SP-ansatz. According to this definition, the indices $k, l$ can only take the values $i, j$ or $j, i$, i.e., the ansatz is "diagonal" in the pair indices. This should be kept in mind when considering the following expressions, in which it is always assumed that $T_{k l}^{i j}$ is restricted in this way.

To demonstrate how the proposed pair-specific geminal ansatz of Eq. (3) can be incorporated into MP2-F12 and CCSD-F12 methods, we will now recapitulate the relevant equations of such methods and show which changes have to be made. In MP2-F12, the conventional amplitdues $T_{a b}^{i j}$ for the double excitations into virtual orbitals are computed as

$$
T_{a b}^{i j}=-\frac{K_{a b}^{i j}+\mathcal{C}_{a b}^{i j}}{\epsilon_{a}+\epsilon_{b}-\epsilon_{i}-\epsilon_{j}},
$$

where $\epsilon_{r}$ are the Hartree-Fock (HF) orbital energies. The MP2-F12 energy is given by the Hylleraas functional

$$
\mathcal{E}_{\mathrm{MP} 2 \mathrm{~F} 12}=\left[\tilde{T}_{a b}^{i j}\left(K_{a b}^{i j}+\mathcal{C}_{a b}^{i j}\right)+2 \mathcal{V}^{i j}+\mathcal{B}^{i j}-\left(\epsilon_{i}+\epsilon_{j}\right) \mathcal{X}^{i j}\right]
$$




\section{Molecular Physics}

with $\tilde{T}_{a b}^{i j}=2 T_{a b}^{i j}-T_{a b}^{j i}$. The matrix elements are defined as

$$
\begin{aligned}
K_{a b}^{i j} & =\left\langle a b\left|r_{12}^{-1}\right| i j\right\rangle, \\
\mathcal{C}_{a b}^{i j} & =\left\langle a b\left|\left(\hat{f}_{1}+\hat{f}_{2}\right) \hat{Q}_{12} F_{12}\right| k l\right\rangle T_{k l}^{i j}, \\
\mathcal{V}^{i j} & =\tilde{T}_{k l}^{i j}\left\langle k l\left|F_{12}^{(i j)} \hat{Q}_{12} r_{12}^{-1}\right| i j\right\rangle, \\
\mathcal{B}^{i j} & =\tilde{T}_{k l}^{i j}\left\langle k l\left|F_{12}^{(i j)} \hat{Q}_{12} \hat{f}_{12} \hat{Q}_{12} F_{12}^{(i j)}\right| m n\right\rangle T_{m n}^{i j}, \\
\mathcal{X}^{i j} & =\tilde{T}_{k l}^{i j}\left\langle k l\left|F_{12}^{(i j)} \hat{Q}_{12} F_{12}^{(i j)}\right| m n\right\rangle T_{m n}^{i j} .
\end{aligned}
$$

where $\hat{f}_{12}=\hat{f}_{1}+f_{2}$ are the Fock operators acting on the electron coordinates 1 and 2 , and $\tilde{T}_{k l}^{i j}=2 T_{k l}^{i j}-T_{k l}^{j i}$. Explicit expressions to evaluate these quantities can be found, for example, in Ref. [26].

In the CCSD-F12a and F12b methods, the standard CCSD singles and doubles residuals $r_{a}^{i}$ and $R_{a b}^{i j}$, respectively, are augmented by a few additional terms

$$
\begin{aligned}
r_{a}^{i, \mathrm{~F} 12 \mathrm{a}} & =r_{a}^{i}+\sum_{k}\left(2 \mathcal{V}_{a k}^{i k}-\mathcal{V}_{a k}^{k i}\right) \\
R_{a b}^{i j, \mathrm{~F} 12 \mathrm{a}} & =R_{a b}^{i j}+\mathcal{V}_{a b}^{i j}+\mathcal{C}_{a b}^{i j}-\mathcal{V}_{a k}^{i j} t_{b}^{k}-t_{a}^{k} \mathcal{V}_{b k}^{i j}
\end{aligned}
$$

where

$$
\mathcal{V}_{r s}^{i j}=\left\langle r s\left|r_{12}^{-1} \hat{Q}_{12} F_{12}^{(i j)}\right| k l\right\rangle T_{k l}^{i j}
$$

In order to simplify the evaluation of the latter quantity, the projector can be approximated by $\hat{Q}_{12}=1-|r s\rangle\langle r s|$, that is, the CABS contributions are neglected[21]. In MOLPRO, this approximation is made by default, even though the full projector can also be used[8]. The CCSD singles and doubles amplitudes $t_{a}^{i}$ and $T_{a b}^{i j}$ are optimized by solving the amplitude equations $r_{a}^{i, \text { F12a }}=0$ and $R_{a b}^{i j, \text { F12a }}=0$. The CCSD-F12a energy is then evaluated in analogy to eq. (8)

$$
\mathcal{E}_{\mathrm{CCSD}-\mathrm{F} 12 \mathrm{a}}=\left[\tilde{D}_{a b}^{i j} K_{a b}^{i j}+\tilde{T}_{a b}^{i j} \mathcal{C}_{a b}^{i j}+2 \mathcal{V}^{i j}+\mathcal{B}^{i j}-\left(\epsilon_{i}+\epsilon_{j}\right) \mathcal{X}^{i j}\right]
$$

where

$$
D_{r s}^{i j}=\sum_{a b} \delta_{r a} \delta_{r b}\left(T_{a b}^{i j}+t_{a}^{i} t_{b}^{j}\right)+\delta_{r i} \sum_{b} \delta_{s b} t_{b}^{j}+\delta_{s j} \sum_{a} \delta_{r a} t_{a}^{i}
$$

and $\tilde{D}_{a b}^{i j}=2 D_{a b}^{i j}-D_{a b}^{j i}$. The CCSD-F12b energy is defined as

$$
\mathcal{E}_{\mathrm{CCSD}-\mathrm{F} 12 \mathrm{~b}}=\mathcal{E}_{\mathrm{CCSD}-\mathrm{F} 12 \mathrm{a}}+\tilde{D}_{r s}^{i j} \mathcal{V}_{r s}^{i j}
$$

In the $\operatorname{CCSD}\left(\mathrm{F} 12^{*}\right)$ approximation[25] some further terms, which involve CABS orbitals, are added to the CCSD residuals. In our spin-free closed-shell formulation 
these read

$$
\begin{aligned}
R_{a b}^{i j,(\mathrm{~F} 12 *)} & =R_{a b}^{i j, \mathrm{~F} 12 \mathrm{a}}+\mathcal{V}_{k l}^{i j} D_{a b}^{k l}-\mathcal{V}_{k}^{i} T_{a b}^{k j}-T_{a b}^{i k} \mathcal{V}_{k}^{j}+U_{a b}^{i j}+U_{b a}^{j i} \\
r_{a}^{i,(\mathrm{~F} 12 *)} & =r_{a}^{i, \mathrm{~F} 12 \mathrm{a}}+u_{a}^{i}+\mathcal{F}_{a x}^{i k} f_{x}^{k}-\mathcal{V}_{k}^{i} t_{a}^{k} \\
U_{a b}^{i j} & =\tilde{\mathcal{F}}_{a x}^{i k}\left(K_{x b}^{k j}-\frac{1}{2} J_{x b}^{k j}\right)-\frac{1}{2} \mathcal{F}_{a x}^{k i} J_{x b}^{k j}-\mathcal{F}_{b x}^{k i} J_{x a}^{k j} \\
u_{a}^{i} & =-\mathcal{F}_{a x}^{k l} l_{x}^{l k i},
\end{aligned}
$$

where $\mathcal{V}_{k}^{i}=\sum_{l} \mathcal{V}_{k l}^{i l}$ and $l_{x}^{k l i}=2 K_{x i}^{k l}-K_{x i}^{l k}$. In this case the full projector is used to compute $\mathcal{V}_{r s}^{i j}$, which actually causes the largest part of the additional computational effort. Furthermore, the integrals $J_{x a}^{k l}=\left\langle k x\left|r_{12}^{-1}\right| l b\right\rangle$ need to be evaluated, which are not required in CCSD-F12a,b.

The $\left(\mathrm{F} 12^{*}\right)$ correction to the energy reads

$$
E_{\mathrm{CCSD}\left(\mathrm{F} 12^{*}\right)}=E_{\mathrm{CCSD}-\mathrm{F} 12 \mathrm{~b}}+U_{a b}^{i j} \tilde{T}_{a b}^{i j}+2 u_{a}^{i} t_{a}^{i}
$$

It should be noted that our $\operatorname{CCSD}\left(\mathrm{F} 12^{*}\right)$ implementation in MOLPRO[30] differs from the $\operatorname{CCSD}\left(\mathrm{F} 12^{*}\right)$ one of Hättig et al.[25] in TURBOMOLE by different density fitting approximations. In TURBOMOLE most integrals classes in the CCSD program are approximated by density fitting, while in MOLPRO they are computed exactly, and only the integrals involving $F_{12}$ or CABS orbitals are density fitted. For this reason the two programs yield exactly the same results only in the limit of very large density fitting basis sets.

For all three CCSD-F12 approximations, the triples $(\mathrm{T})$ energy correction is computed as in standard $\operatorname{CCSD}(\mathrm{T})$. It is not explicitly correlated. While a genuine F12 treatment for (T) has been proposed by Köhn[16], it has not yet been implemented in a production-level program.

Due to the "diagonal" ansatz with canonical orbitals used, the MP2-F12 expressions for different pairs $i j$ are entirely decoupled, and for each pair only one $F_{12}^{(i j)}$ is needed. The $B$ and $X$ matrices are diagonal in the pair indices, i.e., only $B_{i j, i j}$ and $B_{i j, j i}$ are needed. The matrix elements for different $i j$ may involve integrals over different geminals, but there are no integrals needed which involve two different geminals for a single $i j$, and there is only one $B$ and one $X$ matrix. This means that the MP2-F12 and CCSD(T)-F12x formalisms remains completely unchanged, once the integrals have been evaluated. However, the integrals involving $F_{12}^{(i j)}$ have to be computed for each value of $\gamma^{(i j)}$. We use density fitting approximations, and then only two-center and three center integrals are needed. The computational effort for their evaluation and transformation is roughly proportional to the number of different $\gamma^{(i j)}$ values. The most expensive step is the assembly of the 4-index integrals from 3-index quantities, which scales with $\mathcal{O}\left(N^{5}\right)$. The cost for this step is not affected by the pair-specific geminal treatment, since the number of final transformed integrals remains unchanged. Thus, the additional computational effort is small. It mainly depends on the efficiency of the 3-index integral evaluation, which is only a $\mathcal{O}\left(N^{3}\right)$ process.

In the current work a new integral code written by one of us (GK) has been employed. This code, denoted as adaptive integral core (AIC), is overall around 5-10 times faster for F12 integrals than our previous one[31, 32]. For the integration, the F12 Slater geminal of eq. (3) is approximated by a linear combination of six Gaussian geminals[26]. The integrals are evaluated with a variant of the ObaraSaika scheme[33-35] in Ahlrichs' three-center solid harmonic modification[36]. The 


\section{Molecular Physics}

integral kernel functions $G_{m}(\rho, T)$ necessary for contracted Gaussian geminals are documented in Ref. [37]. For some F12 integral classes, up to $70 \%$ of the total integration time are spent evaluating the scalar $G_{m}(\rho, T)$ kernels, and in these cases some computational savings (up to around 20\%) are possible by employing generally contracted Gaussian geminals with shared exponents. These are supported in the new integral code. In this work, however, the Slater geminals are fitted independently to six Gaussians each, as described in Ref. [26], with non-shared exponents. In this case the cost of the raw F12 integral evaluation rises in proportion to the number of exponents used.

In order to keep the integral evaluation cost to a minimum, we only distinguish core-core, core-valence, and valence-valence pairs. One can either use three different geminal exponents for these classes, or treat core-core and core-valence pairs with the same geminal exponent. As will be shown later this is justified, since the core-core pairs usually have a negligible effect on energy differences and molecular properties.

A final note concerns the use of non-canonical orbitals or open-shell cases using spin-restricted Hartree-Fock (RHF) reference functions. In these cases the Fock matrix is not diagonal any more, and the MP2-F12 equations have to be solved iteratively $[8,26]$. In the energy expression of Eq. (17), only the term containing eigenvalues needs to be modified, according to

$$
-\left(\epsilon_{i}+\epsilon_{j}\right) \mathcal{X}^{i j} \rightarrow-\left(\mathcal{X}_{i k}^{i j} f_{k j}+f_{i k} \mathcal{X}_{k j}^{i j}\right)
$$

with

$$
\mathcal{X}_{k l}^{i j}=T_{m n}^{i j}\left\langle m n\left|F_{12}^{(i j)} \hat{Q}_{12} F_{12}^{(k l)}\right| o p\right\rangle T_{o p}^{k l}
$$

Using the projector in eq. (5) the matrix elements are evaluated as

$$
\begin{aligned}
\left\langle m n\left|F_{12}^{(i j)} \hat{Q}_{12} F_{12}^{(k l)}\right| o p\right\rangle= & \left\langle m n\left|F_{12}^{(i j)} F_{12}^{(k l)}\right| o p\right\rangle \\
& -\left\langle m n\left|F_{12}^{(i j)}\right| r s\right\rangle\left\langle r s\left|F_{12}^{(k l)}\right| o p\right\rangle \\
& -\left\langle m n\left|F_{12}^{(i j)}\right| i x\right\rangle\left\langle i x\left|F_{12}^{(k l)}\right| o p\right\rangle \\
& -\left\langle m n\left|F_{12}^{(i j)}\right| x i\right\rangle\left\langle x i\left|F_{12}^{(k l)}\right| o p\right\rangle .
\end{aligned}
$$

Thus, in this case we need the integrals $\left\langle m n\left|F_{12}^{(i j)} F_{12}^{(k l)}\right| o p\right\rangle$ involving two different geminals. Using robust density fitting these are computed as[31, 38]

$$
\begin{aligned}
\left\langle m n\left|F_{12}^{(i j)} F_{12}^{(k l)}\right| o p\right\rangle= & \left(m n\left|F_{12}^{(i j)} F_{12}^{(k l)}\right| A\right) d_{o p}^{A}+d_{m n}^{A}\left(o p\left|F_{12}^{(i j)} F_{12}^{(k l)}\right| A\right) \\
& -d_{m n}^{A}\left\langle A\left|F_{12}^{(i j)} F_{12}^{(k l)}\right| B\right\rangle d_{o p}^{B},
\end{aligned}
$$

where $d_{m n}^{A}$ are the fitting coefficients obtained by solving the linear equations

$$
\left(A\left|r_{12}^{-1}\right| B\right) d_{m n}^{B}=\left(m n\left|r_{12}^{-1}\right| A\right)
$$


and the integrals for a kernel $\hat{O}$ are defined as

$$
\begin{aligned}
(A|\hat{O}| B) & =\int \mathrm{d} \mathbf{r}_{1} \int \mathrm{d} \mathbf{r}_{2} \chi_{A}\left(\mathbf{r}_{1}\right) \hat{O} \chi_{B}\left(\mathbf{r}_{2}\right) \\
(m n|\hat{O}| A) & =\int \mathrm{d} \mathbf{r}_{1} \int \mathrm{d} \mathbf{r}_{2} \chi_{m}\left(\mathbf{r}_{1}\right) \chi_{n}\left(\mathbf{r}_{1}\right) \hat{O} \chi_{A}\left(\mathbf{r}_{2}\right) .
\end{aligned}
$$

Note that the fitting coefficients are independent of the geminal exponent and need to be computed only once.

The method described above is orbital-invariant within the core, closed-shell valence, and open-shell valence subspaces. Of course, due to the different geminal exponents, it is not invariant with respect to orbital rotations between the core and valence orbitals.

\section{Applications}

In order to test the method, we have computed energies and spectroscopic constants for a number of diatomic molecules, namely $\mathrm{Br}_{2}\left(\mathrm{X}^{1} \Sigma_{g}^{+}\right), \mathrm{As}_{2}\left(\mathrm{X}^{1} \Sigma_{g}^{+}\right), \mathrm{Ga}_{2}$ $\left(\mathrm{X}^{3} \Pi_{u}\right), \mathrm{Cu}_{2}\left(\mathrm{X}^{1} \Sigma_{g}^{+}\right), \mathrm{GaCl}\left(\mathrm{X}^{1} \Sigma^{+}\right), \mathrm{CuCl}\left(\mathrm{X}^{1} \Sigma^{+}\right)$, and $\mathrm{CuBr}\left(\mathrm{X}^{1} \Sigma^{+}\right)$. In all these cases core-valence correlation effects are significant, in particular on the equilibrium bond lengths. Here we define "core" to be the $3 d$ orbitals; correlation of the $3 s$ and $3 p$ orbitals in $\mathrm{Br}, \mathrm{As}, \mathrm{Ga}$, and $\mathrm{Cu}$ has a very small effect on the results and has therefore not been included. For Br, As, and Ga we used newly optimized cc-pVnZ-F12-PP orbital basis sets of Peterson and Hill[39], along with their associated OPTRI sets for CABS construction and MP2FIT sets for density fitting. All OPTRI basis sets include basis functions up to $l=6(i)$, as required to treat the correlation of $d$-electrons. However, the relative energies were found to be rather insensitive to the high angular momentum functions, and 1-2 $i$ functions are sufficient (the VDZ-F12/OPTRI sets contain one $i$, the VTZ-F12/OPTRI and VQZF12/OPTRI sets two $i$ functions). Similar basis sets for treating core-valence correlation effects were recently published by Hill et al. for lighter atoms[40]. For simplicitly, these basis sets will be denoted VnZ-F12 in the following. For $\mathrm{Cu}$ we used the aug-cc-pVnZ-PP basis[41] sets along with their OPTRI counterparts[42]. In this case Hättig's MP2FIT sets[43] were used. Finally, for $\mathrm{Cl}$ the aug-cc-pV $(n+d) \mathrm{Z}$ OBSs (with additional steep $d$-functions[44]) and the corresponding OPTRI basis sets[45] were employed. Robust density fitting approximations[31, 32] were used to compute all $F_{12}$-dependent integrals, as well as all other integrals involving CABS orbitals. In all cases the Fock matrix in the combined OBS/CABS basis, which is needed in MP2-F12, was density fitted using the QZVPP/JKFIT basis sets of Weigend[46]. All other integrals that occur in the MP2-F12 and CCSDF12x equations (including the Fock matrix in the OBS) were computed exactly without density fitting. The small-core pseudopotentials (ECP10MDF) of Stoll, Dolg and co-workers[47-49] were used for the heavy atoms (not for $\mathrm{Cl}$ ). Since the pseudopotentials do not commute with the correlation factor, they were treated in the MP2-F12 exactly as the exchange contribution, as noted by Bischoff et al. [50]. In the open-shell coupled-cluster calculations we used the partially spin-restricted RCCSD(T) method[8, 51], using spin restricted open-shell Hartree-Fock reference functions.

In the case of $\mathrm{GaCl}$, a slight complication arises because the $\mathrm{Cl}(3 s)$ orbital mixes significantly with the Ga $\left(3 d_{0}\right)$ one. This causes poor results if either just the valence orbitals (without $3 d$ ) are correlated, or when valence and $3 d$ orbitals 

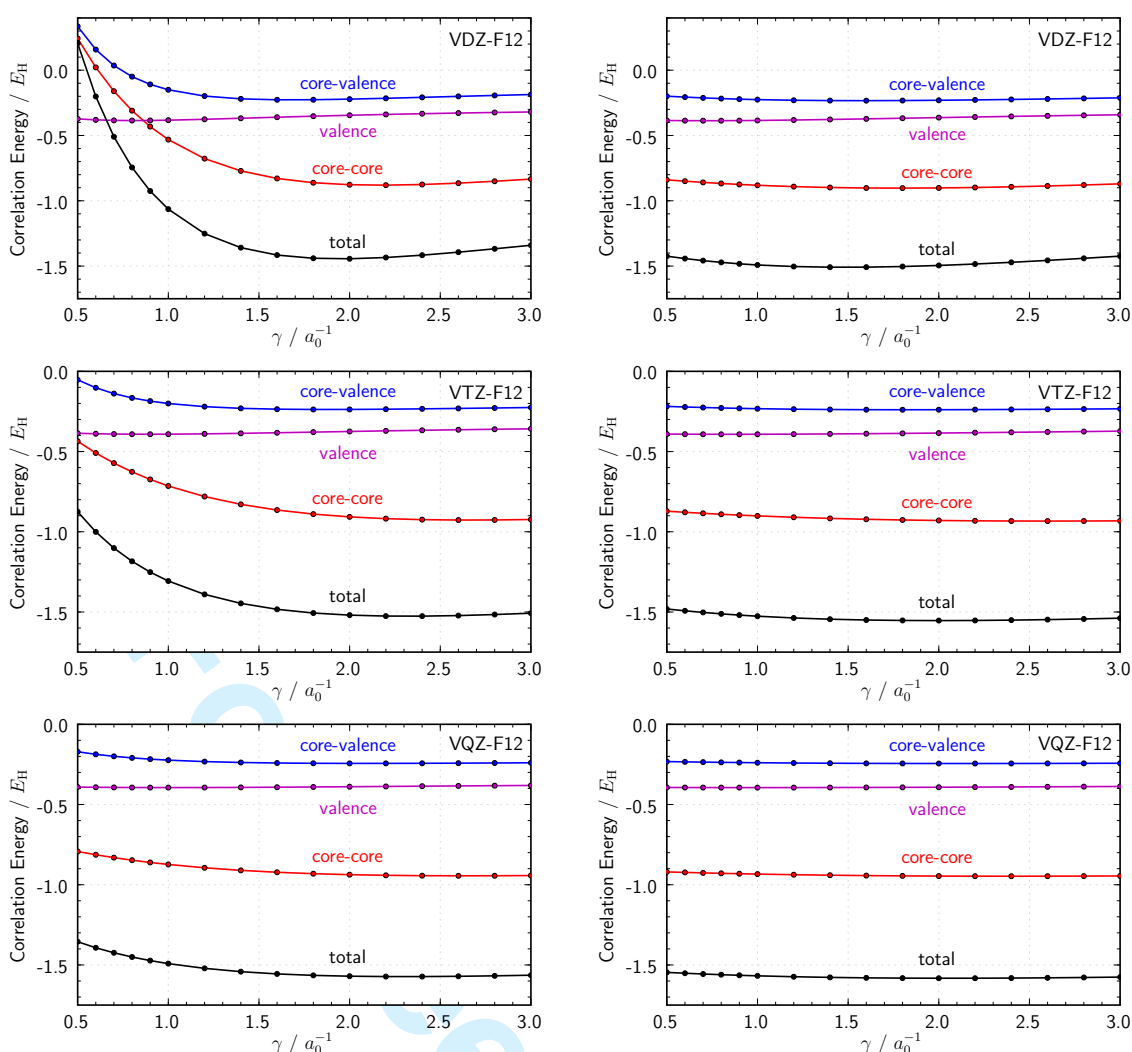

(a) Using the fixed amplitude approximation (b) Using optimized geminal amplitudes (diagonal ansatz)

Figure 1. Dependence of the MP2-F12 core-core, core-valence and valence energy contributions of the correlation energy of $\operatorname{Br}_{2}(r=2.281 \mathrm{~A})$ on the parameter $\gamma$.

are treated with different geminal exponents. This problem can be avoided by a $2 \times 2$ Boys localization of the $\mathrm{Cl}(3 s)$ and $\mathrm{Ga}\left(3 d_{0}\right)$ orbitals. One must then use the orbital invariant form of the MP2-F12 equations (c.f. previous section). Before computing the perturbative $(\mathrm{T})$ triples correction, the orbitals are re-canonicalized, and the CCSD amplitudes are transformed accordingly. A similar problem occurs for $\mathrm{CuCl}$ and $\mathrm{CuBr}$, but in these cases the $\mathrm{Cl}(3 p)$ and $\mathrm{Ga}(3 d)$ orbitals mix. Again, localization was used to reduce the mixing as much as possible.

We will first consider the core-core $(c c)$, core-valence $(c v)$ and valence $(v v)$ correlation contributions as function of the geminal exponent. A similar analysis has been performed by Bischoff et al. at the level of MP2-F12 theory[50]. Since the MP2-F12 pair energies are uncoupled, the exponents for the different classes can be optimized independently (in the cases with localized orbitals and in the open-shell case of $\mathrm{Ga}_{2}$ this is only approximately true). The dependence of the correlation energy contributions on the geminal exponent is shown for $\mathrm{Br}_{2}$ in Fig. 1.

It is strongest for the $c c$ energy contribution. For small exponents $\gamma$ and the cc-pVDZ-F12 basis set, the energy contribution even becomes positive. This is a consequence of the fixed amplitude approximation and the fact that the energy is computed using the variational Hylleraas functional. The dependence on $\gamma$ is very much reduced if the geminal coefficients $T_{i j}^{i j}$ and $T_{j i}^{i j}$ are optimized, as is demonstrated in Fig. 1. This optimization is easy at the MP2-F12 level, but difficult and expensive for CCSD-F12. One way out is to use the optimized MP2-F12 amplitudes as fixed parameters in the CCSD-F12. Even though this approximation is more pragmatic than well justified, it works quite well, as will be shown in another forthcoming publication[52]. In the current work we will stick to the fixed 
Table 1. Comparison of MP2-F12 correlation energy contributions for $\mathrm{Br}_{2}$, $\mathrm{r}=2.281 \mathrm{~A}$

\begin{tabular}{lrrrr}
\hline$\beta^{a}$ & $E_{\mathrm{cc}}$ & $E_{\mathrm{cv}}$ & $E_{\mathrm{vv}}$ & $E_{\mathrm{corr}}$ \\
\hline cc-pVDZ-F12: & & & & \\
none & -0.348225 & -0.106380 & -0.286396 & -0.741001 \\
1.25 & -0.704926 & -0.205413 & -0.374249 & -1.284588 \\
$0.8,1.7$ & -0.847970 & -0.227079 & -0.385674 & -1.460722 \\
$0.8,1.7,2.2$ & -0.880350 & -0.227079 & -0.385674 & -1.493103 \\
& & & & \\
cc-pVTZ-F12: & & & & \\
none & -0.615204 & -0.174067 & -0.330602 & -1.119874 \\
1.4 & -0.829053 & -0.230702 & -0.386580 & -1.446335 \\
$0.9,1.9$ & -0.899375 & -0.237835 & -0.392111 & -1.529321 \\
$0.9,1.9,2.6$ & -0.926908 & -0.237835 & -0.392111 & -1.556854 \\
cc-pVQZ-F12: & & & & \\
none & & & & \\
1.4 & -0.758205 & -0.207960 & -0.362500 & -1.328665 \\
$1.0,2.1$ & -0.910334 & -0.238061 & -0.393400 & -1.541795 \\
$1.0,2.1,2.7$ & -0.939559 & -0.243764 & -0.394562 & -1.577885 \\
\hline
\end{tabular}

aThe first, second, and third values are used for $v v, c v, c c$ pairs, respectively. If less than 3 values are given, the last value is also used for the missing ones.

amplitude ansatz.

In Tables 1-4 we present the correlation energies computed with up to 3 different geminal exponents for the $v v, c v$, and $c c$ pairs, respectively. In case with 2 exponents, the $c c$ and $c v$ pairs are treated with the same exponent. The exponents were roughly optimized for each class by running MP2-F12 calculations for a range of $\gamma$ values between 0.5 and $3.0 a_{0}^{-1}$, as shown in Fig. 1 for $\mathrm{Br}_{2}$. If only a single exponent is used for all pairs, an average value for the $v v$ and $c v$ is employed. Note that the chosen values do not maximize the total correlation energy, which is dominated by the $c c$ contribution. The latter does not have a large effect on the molecular properties, and it is therefore more important to choose appropriate geminals for the $v v$ and $c v$ contributions. An exception is $\mathrm{Cu}_{2}$. Due to the diffuse nature of the $4 s$ "valence" orbital the corresponding optimized exponent is very small. If only a single geminal exponent is used, the results for the spectroscopic constants are rather sensitive to its choice, and we found that larger exponents, which are more appropriate for correlation of the $d$-shell, improve the results. For simplicity, we used the exponent optimized for the $c v$ energy contribution in this case.

The results show the expected trends: The optimized geminal exponents are smallest for the $v v$ and largest for the $c c$ correlation contributions. They increase with increasing nuclear charge, i.e. from left to right in the periodic table. They also increase with increasing OBS, since for large basis sets the role of the geminals is mainly to improve the cusp region for small $r_{12}$, while for small basis sets they also help to model a larger range of the correlation hole. We note that the geminal exponents should not be optimized using the CCSD-F12x approximations. Since in these methods an approximate Lagrangian is used to compute the energy, which is not variational as the Hylleraas functional for MP2-F12, artificial results may result.

The effect of the explicit correlation treatment on the correlation energies is quite dramatic, in particular for the double- $\zeta$ basis set. For $\mathrm{Br}_{2}$ and $\mathrm{As}_{2}$ the standard MP2 with the VDZ-F12 basis set recovers less than $40 \%$ of the $c c$ correlation energies. This is due to the fact that these bases were not devised for $c v$ and $c c$ correlation without including F12; they contain only very few additional functions for correlating the $d$-shell. For a proper treatment of $c c$ and $c v$ effects without F12, the much larger aug-cc-pwCVnZ-PP basis sets[53] would be needed. In contrast 
Table 2. Comparison of MP2-F12 correlation energy contributions for $\mathrm{As}_{2}$, $\mathrm{r}=2.094 \mathrm{~A}$

\begin{tabular}{lrrrr}
\hline$\beta^{a}$ & $E_{\mathrm{cc}}$ & $E_{\mathrm{cv}}$ & $E_{\mathrm{vv}}$ & $E_{\mathrm{corr}}$ \\
\hline cc-pVDZ-F12: & & & & \\
none & -0.365514 & -0.098149 & -0.237425 & -0.701089 \\
1.20 & -0.761724 & -0.172542 & -0.282661 & -1.216927 \\
$0.75,1.50$ & -0.862881 & -0.181837 & -0.290339 & -1.335056 \\
$0.75,1.50,2.0$ & -0.904589 & -0.181837 & -0.290339 & -1.376764 \\
& & & & \\
cc-pVTZ-F12: & & & & \\
none & -0.569346 & -0.138765 & -0.262744 & -0.970856 \\
0.2 & -0.796320 & -0.179895 & -0.291208 & -1.267423 \\
$0.8,1.6,2.2$ & -0.895576 & -0.188822 & -0.293300 & -1.377698 \\
cc-pVQZ-F12: & -0.936038 & -0.188822 & -0.293300 & -1.418160 \\
none & & & & \\
1.4 & -0.788728 & -0.171469 & -0.277584 & -1.237780 \\
$1.0,2.1$ & -0.927424 & -0.191992 & -0.293235 & -1.412650 \\
$1.0,2.1,2.7$ & -0.953087 & -0.194372 & -0.294394 & -1.441854 \\
\hline
\end{tabular}

a The first, second, and third values are used for $v v, c v, c c$ pairs, respectively. If less than 3 values are given, the last value is also used for the missing ones.

Table 3. Comparison of MP2-F12 correlation energy contributions for $\mathrm{Ga}_{2}$, $\mathrm{r}=2.65 \mathrm{~A}$

\begin{tabular}{lrrrr}
\hline$\beta^{a}$ & $E_{\mathrm{cc}}$ & $E_{\mathrm{cv}}$ & $E_{\mathrm{vv}}$ & $E_{\mathrm{corr}}$ \\
\hline cc-pVDZ-F12: & & & & \\
none & -0.436434 & -0.076403 & -0.087732 & -0.600570 \\
1.00 & -0.758908 & -0.123747 & -0.100080 & -0.982734 \\
$0.7,1.30$ & -0.892358 & -0.132306 & -0.101460 & -1.126123 \\
$0.7,1.3,1.75$ & -0.939597 & -0.132305 & -0.101460 & -1.173363 \\
& & & & \\
cc-pVTZ-F12: & & & & \\
none & -0.613708 & -0.1075658 & -0.094895 & -0.816168 \\
1.1 & -0.843933 & -0.133778 & -0.102076 & -1.079786 \\
$0.8,1.40$ & -0.930319 & -0.138097 & -0.102507 & -1.170924 \\
$0.8,1.4,2.0$ & -0.974577 & -0.138097 & -0.102507 & -1.215183 \\
& & & & \\
cc-pVQZ-F12: & & & & \\
none & -0.771407 & -0.126497 & -0.098796 & -0.996700 \\
1.3 & -0.941749 & -0.140226 & -0.102655 & -1.184630 \\
$0.9,1.7$ & -0.982998 & -0.141579 & -0.102879 & -1.227457 \\
$0.9,1.7,2.3$ & -0.998691 & -0.141579 & -0.102879 & -1.243150 \\
\hline
\end{tabular}

aThe first, second, and third values are used for $v v, c v, c c$ pairs, respectively. If less than 3 values are given, the last value is also used for the missing ones.

to standard MP2, the MP2-F12 method recovers with just one geminal exponent about $75-80 \%$ and with the optimized exponent for core correlation $93-94 \%$ of the $c c$ correlation energies, using the small VDZ-F12 basis (as compared to the best MP2-F12/VQZ-F12 energies). For $\mathrm{Cu}_{2}$ the effect is less dramatic, since the aug-cc-pVnZ-PP basis set used in this case includes functions for $d$-correlation. Nevertheless, there is still a significant improvement by the F12-treatment, and using optimized geminal exponents for $v v, c v$, and $v v$ leads to quite impressive energy lowerings. Of course, these effects become less pronounced for the larger triple- $\zeta$ and quadruple- $\zeta$ basis sets.

The computed equilibrium distances, harmonic and anharmonic vibrational frequencies $\omega_{e}$ as well as dissociation energies $D_{e}$ of the homonuclear diatomics are presented in Tables 5-8. These were obtained from polynomial fits of 8 th degree to 9 points in the range $r_{e}-0.4 \mathrm{~A}$ to $r_{e}+0.7 \mathrm{~A}$. The core-valence correlation effects reduce the equilibrium distances and increase the harmonic wavenumbers significantly. As for the energies, the convergence with the basis set size is slow without 
Table 4. Comparison of MP2-F12 correlation energy contributions for $\mathrm{Cu}_{2}$, $\mathrm{r}=2.22 \mathrm{~A}$

\begin{tabular}{lrrrr}
\hline$\beta^{a}$ & $E_{\mathrm{cc}}$ & $E_{\mathrm{cv}}$ & $E_{\mathrm{vv}}$ & $E_{\mathrm{corr}}$ \\
\hline cc-pVDZ-F12: & & & & \\
none & -0.897994 & -0.066005 & -0.018568 & -0.982567 \\
1.0 & -1.040207 & -0.079914 & -0.020312 & -1.140433 \\
$0.55,1.0$ & -1.040207 & -0.079914 & -0.020612 & -1.140732 \\
$0.55,1.0,1.9$ & -1.108529 & -0.079914 & -0.020612 & -1.209055 \\
cc-pVTZ-F12: & & & & \\
none & -1.020649 & -0.077546 & -0.019367 & -1.117562 \\
1.0 & -1.104856 & -0.083657 & -0.020562 & -1.209076 \\
$0.55,1.0$ & -1.104856 & -0.083657 & -0.020753 & -1.209266 \\
$0.55,1.0,2.0$ & -1.127407 & -0.083657 & -0.020753 & -1.231817 \\
cc-pVQZ-F12: & & & & \\
none & & & & \\
1.0 & -1.081396 & -0.081754 & -0.019867 & -1.183016 \\
$0.55,1.0$ & -1.127094 & -0.085079 & -0.020692 & -1.232865 \\
$0.55,1.0,2.2$ & -1.127094 & -0.085079 & -0.020811 & -1.232983 \\
\hline
\end{tabular}

aThe first, second, and third values are used for $v v, c v, c c$ pairs, respectively. If less than 3 values are given, the last value is also used for the missing ones.

Table 5. Computed CCSD(T)-F12b spectroscopic constants for $\mathrm{Br}_{2}$ with different numbers of geminals. The geminal exponents are the same as in Table 1. $r_{e}$ in $\mathrm{A} \omega_{e}$ and $\omega_{e} x_{e}$ in $\mathrm{cm}^{-1}, D_{e}$ in $\mathrm{eV}$.

\begin{tabular}{lccccc}
\hline Basis & Geminals & $r_{e}$ & $\omega_{e}$ & $\omega_{e} x_{e}$ & $D_{e}$ \\
\hline VDZ-F12 & 0 & 2.3211 & 305.6 & 1.02 & 1.88 \\
VTZ-F12 & 0 & 2.2994 & 320.6 & 1.04 & 2.09 \\
VQZ-F12 & 0 & 2.2884 & 325.8 & 1.00 & 2.19 \\
VDZ-F12 & 1 & 2.2815 & 326.8 & 1.02 & 2.19 \\
VTZ-F12 & 1 & 2.2806 & 328.0 & 1.03 & 2.22 \\
VQZ-F12 & 1 & 2.2775 & 330.1 & 1.01 & 2.26 \\
VDZ-F12 & 2 & 2.2756 & 331.0 & 1.02 & 2.26 \\
VTZ-F12 & 2 & 2.2790 & 328.7 & 1.02 & 2.24 \\
VQZ-F12 & 2 & 2.2767 & 330.4 & 1.01 & 2.26 \\
& & & & & \\
VDZ-F12 & 3 & 2.2758 & 330.9 & 1.02 & 2.26 \\
VTZ-F12 & 3 & 2.2789 & 328.7 & 1.02 & 2.24 \\
VQZ-F12 & 3 & 2.2767 & 330.4 & 1.01 & 2.26 \\
aug-wCV[Q5]Z-PP & 0 & 2.2740 & 331.2 & 0.99 & 2.23 \\
Experiment & & & & & \\
\hline
\end{tabular}

${ }^{\mathrm{a}} D_{e}$ value corrected for spin-orbit effect

explicit correlation (denoted 0 geminals in the tables), and very much improved by the F12 treatment. Taking $\mathrm{Br}_{2}$ as an example, the standard CCSD(T) calculation with VDZ-F12 basis yields a distance that is $0.047 \mathrm{~A}$ too long. This error is reduced by the F12-treatment to 0.008 and $0.002 \mathrm{~A}$ with 1 or 2 geminal exponents, respectively. Using a separate exponent for the $c c$ correlation has only a very minor effect. Already with a single geminal exponent the F12 values with VDZ-F12 are better than the conventional ones with VQZ-F12 basis. Further increase of the basis set to VQZ-F12 leads only to a shortening by $0.001 \mathrm{~A}$. The effects are qualitatively similar for the other molecules. For $\mathrm{Cu}_{2}$, where the OBS is designed to include $d$-correlation effects, the improvements by the F12 terms are somewhat smaller, but there is still a significant effect, in particular for the smaller basis sets. Overall, the results clearly show that using an optimized geminal exponent for $c v$ correlation leads to an improvement, while the third exponent for $c c$ correlation has only a minor effect.

A significant improvement by the F12 treatment is also found for the vibrational 


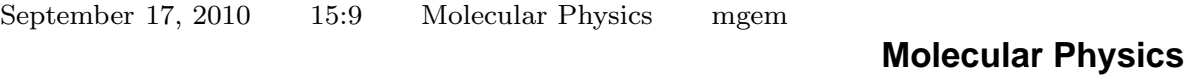

Table 6. Computed CCSD(T)-F12b spectroscopic constants for $\mathrm{As}_{2}$ with different numbers of geminals. The geminal exponents are the same as in Table 2. $r_{e}$ in $\mathrm{A} \omega_{e}$ and $\omega_{e} x_{e}$ in $\mathrm{cm}^{-1}, D_{e}$ in $\mathrm{eV}$

\begin{tabular}{lccccr}
\hline Basis & Geminals & $r_{e}$ & $\omega_{e}$ & $\omega_{e} x_{e}$ & $D_{e}$ \\
\hline VDZ-F12 & 0 & 2.1129 & 420.4 & 1.04 & 3.33 \\
VTZ-F12 & 0 & 2.1089 & 425.8 & 0.97 & 3.64 \\
VQZ-F12 & 0 & 2.0987 & 432.3 & 0.96 & 3.84 \\
& & & & & \\
VDZ-F12 & 1 & 2.0943 & 435.7 & 1.03 & 3.81 \\
VTZ-F12 & 1 & 2.0963 & 434.9 & 0.99 & 3.91 \\
VQZ-F12 & 1 & 2.0921 & 438.3 & 0.98 & 3.98 \\
& & & & & \\
VDZ-F12 & 2 & 2.0937 & 437.2 & 1.02 & 3.86 \\
VTZ-F12 & 2 & 2.0945 & 436.2 & 0.99 & 3.92 \\
VQZ-F12 & 2 & 2.0917 & 438.7 & 0.98 & 3.98 \\
& & & & & \\
VDZ-F12 & 3 & 2.0944 & 436.7 & 1.01 & 3.86 \\
VTZ-F12 & 3 & 2.0951 & 435.9 & 0.99 & 3.92 \\
VQZ-F12 & 3 & 2.0918 & 438.6 & 0.98 & 3.98 \\
aug-wCV[Q5]Z-PP & 0 & 2.0902 & 440.5 & 0.99 & 4.03 \\
Experiment & & 2.1026 & 429.6 & 1.12 & 3.99 \\
\hline
\end{tabular}

Table 7. Computed CCSD(T)-F12b spectroscopic constants for $\mathrm{Ga}_{2}$ with different numbers of geminals. The geminal exponents are the same as in Table $3 . r_{e}$ in A $\omega_{e}$ and $\omega_{e} x_{e}$ in $\mathrm{cm}^{-1}, D_{e}$ in $\mathrm{eV}$.

\begin{tabular}{lccccc}
\hline Basis & Geminals & $r_{e}$ & $\omega_{e}$ & $\omega_{e} x_{e}$ & $D_{e}$ \\
\hline aug-cc-pVDZ-PP & 0 & 2.6798 & 164.5 & 0.58 & 1.22 \\
aug-cc-pVTZ-PP & 0 & 2.6751 & 169.6 & 0.57 & 1.30 \\
aug-cc-pVQZ-PP & 0 & 2.6561 & 172.7 & 0.59 & 1.34 \\
aug-cc-pVDZ-PP & 1 & 2.6556 & 172.2 & 0.66 & 1.27 \\
aug-cc-pVTZ-PP & 1 & 2.6533 & 173.4 & 0.59 & 1.33 \\
aug-cc-pVQZ-PP & 1 & 2.6480 & 174.2 & 0.59 & 1.35 \\
aug-cc-pVDZ-PP & 2 & 2.6499 & 172.9 & 0.63 & 1.30 \\
aug-cc-pVTZ-PP & 2 & 2.6521 & 173.2 & 0.59 & 1.33 \\
aug-cc-pVQZ-PP & 2 & 2.6483 & 174.0 & 0.59 & 1.35 \\
aug-cc-pVDZ-PP & 3 & 2.6521 & 172.5 & 0.63 & 1.30 \\
aug-cc-pVTZ-PP & 3 & 2.6543 & 173.0 & 0.59 & 1.33 \\
aug-cc-pVQZ-PP & 3 & 2.6492 & 173.9 & 0.59 & 1.35 \\
aug-wCV[Q5]Z-PP & 0 & 2.6464 & 174.4 & 0.57 & 1.36 \\
\hline
\end{tabular}

Table 8. Computed CCSD(T)-F12b spectroscopic constants for $\mathrm{Cu}_{2}$ with different numbers of geminals. The geminal exponents are the same as in Table 4. $r_{e}$ in $\mathrm{A} \omega_{e}$ and $\omega_{e} x_{e}$ in $\mathrm{cm}^{-1}, D_{e}$ in $\mathrm{eV}$.

\begin{tabular}{lccccc}
\hline Basis & Geminals & $r_{e}$ & $\omega_{e}$ & $\omega_{e} x_{e}$ & $D_{e}$ \\
\hline aug-cc-pVDZ-PP & 0 & 2.2357 & 258.1 & 0.94 & 1.93 \\
aug-cc-pVTZ-PP & 0 & 2.2213 & 266.1 & 1.04 & 1.97 \\
aug-cc-pVQZ-PP & 0 & 2.2166 & 266.7 & 1.03 & 2.00 \\
& & & & & \\
aug-cc-pVDZ-PP & 1 & 2.2222 & 264.5 & 1.00 & 2.01 \\
aug-cc-pVTZ-PP & 1 & 2.2162 & 267.9 & 1.03 & 2.01 \\
aug-cc-pVQZ-PP & 1 & 2.2146 & 267.8 & 1.02 & 2.02 \\
aug-cc-pVDZ-PP & 2 & 2.2227 & 264.4 & 1.00 & 2.00 \\
aug-cc-pVTZ-PP & 2 & 2.2162 & 267.9 & 1.03 & 2.01 \\
aug-cc-pVQZ-PP & 2 & 2.2145 & 267.9 & 1.02 & 2.02 \\
aug-cc-pVDZ-PP & 3 & 2.2203 & 263.0 & 1.00 & 1.99 \\
aug-cc-pVTZ-PP & 3 & 2.2159 & 267.1 & 1.03 & 2.00 \\
aug-cc-pVQZ-PP & 3 & 2.2143 & 267.4 & 1.03 & 2.01 \\
aug-cc-pV[Q5]Z-PP & 0 & 2.2146 & 268.6 & 1.01 & 2.03 \\
Experiment & & 2.2197 & 264.6 & 1.02 & \\
\hline
\end{tabular}


Table 9. Computed spectroscopic constants of homonuclear diatomics using different $\operatorname{CCSD}(\mathrm{T})-\mathrm{F} 12 \mathrm{x}$ approximations with 3 geminals (see text) $\omega_{e}$ and $\omega_{e} x_{e}$ in $\mathrm{cm}^{-1}$.

\begin{tabular}{|c|c|c|c|c|}
\hline Basis & Method & $R_{e} / \mathrm{A}$ & $\omega_{e}$ & $\omega_{e} x_{e}$ \\
\hline \multicolumn{5}{|l|}{$\mathrm{Br}_{2}$ : } \\
\hline cc-pVDZ-F12 & $\mathrm{F} 12 \mathrm{a}$ & 2.2764 & 330.7 & 1.02 \\
\hline cc-pVTZ-F12 & $\mathrm{F} 12 \mathrm{a}$ & 2.2793 & 328.8 & 1.02 \\
\hline cc-pVQZ-F12 & $\mathrm{F} 12 \mathrm{a}$ & 2.2767 & 330.5 & 1.01 \\
\hline cc-pVDZ-F12 & $\mathrm{F} 12 \mathrm{~b}$ & 2.2758 & 330.9 & 1.02 \\
\hline cc-pVTZ-F12 & $\mathrm{F} 12 \mathrm{~b}$ & 2.2794 & 328.5 & 1.02 \\
\hline cc-pVQZ-F12 & $\mathrm{F} 12 \mathrm{~b}$ & 2.2769 & 330.3 & 1.01 \\
\hline cc-pVDZ-F12 & $\left(\mathrm{F} 12^{*}\right)$ & 2.2759 & 333.2 & 0.98 \\
\hline cc-pVTZ-F12 & $\left(\mathrm{F} 12^{*}\right)$ & 2.2766 & 331.7 & 1.00 \\
\hline cc-pVQZ-F12 & $\left(\mathrm{F} 12^{*}\right)$ & 2.2752 & 332.0 & 1.00 \\
\hline \multicolumn{2}{|l|}{ aug-wCV[Q5]Z-PP } & 2.2740 & 331.2 & 0.99 \\
\hline \multicolumn{5}{|l|}{$\mathrm{As}_{2}$ : } \\
\hline cc-pVDZ-F12 & $\mathrm{F} 12 \mathrm{a}$ & 2.0935 & 437.5 & 1.01 \\
\hline cc-pVTZ-F12 & $\mathrm{F} 12 \mathrm{a}$ & 2.0941 & 436.9 & 0.98 \\
\hline cc-pVQZ-F12 & $\mathrm{F} 12 \mathrm{a}$ & 2.0915 & 439.0 & 0.98 \\
\hline cc-pVDZ-F12 & $\mathrm{F} 12 \mathrm{~b}$ & 2.0939 & 436.9 & 1.02 \\
\hline cc-pVTZ-F12 & $\mathrm{F} 12 \mathrm{~b}$ & 2.0949 & 436.0 & 0.99 \\
\hline cc-pVQZ-F12 & $\mathrm{F} 12 \mathrm{~b}$ & 2.0920 & 438.6 & 0.98 \\
\hline cc-pVDZ-F12 & $\left(\mathrm{F} 12^{*}\right)$ & 2.0909 & 442.6 & 0.97 \\
\hline cc-pVTZ-F12 & $\left(\mathrm{F} 12^{*}\right)$ & 2.0921 & 440.4 & 0.96 \\
\hline cc-pVQZ-F12 & $\left(\mathrm{F} 12^{*}\right)$ & 2.0905 & 440.6 & 0.96 \\
\hline aug-wCV[Q5]Z-PP & & 2.0902 & 440.5 & 0.99 \\
\hline \multicolumn{5}{|l|}{$\mathrm{Cu}_{2}$ : } \\
\hline aug-cc-pVDZ-PP & $\mathrm{F} 12 \mathrm{a}$ & 2.2159 & 264.0 & 1.00 \\
\hline aug-cc-pVTZ-PP & $\mathrm{F} 12 \mathrm{a}$ & 2.2142 & 267.1 & 1.03 \\
\hline aug-cc-pVQZ-PP & $\mathrm{F} 12 \mathrm{a}$ & 2.2135 & 266.6 & 1.02 \\
\hline aug-cc-pVDZ-PP & $\mathrm{F} 12 \mathrm{~b}$ & 2.2181 & 262.7 & 1.01 \\
\hline aug-cc-pVTZ-PP & $\mathrm{F} 12 \mathrm{~b}$ & 2.2149 & 266.5 & 1.03 \\
\hline aug-cc-pVQZ-PP & $\mathrm{F} 12 \mathrm{~b}$ & 2.2138 & 266.4 & 1.02 \\
\hline aug-cc-pVDZ-PP & $\left(\mathrm{F} 12^{*}\right)$ & 2.2201 & 261.5 & 1.00 \\
\hline aug-cc-pVTZ-PP & $\left(\mathrm{F} 12^{*}\right)$ & 2.2159 & 266.0 & 1.02 \\
\hline aug-cc-pVQZ-PP & $\left(\mathrm{F} 12^{*}\right)$ & 2.2143 & 266.1 & 1.02 \\
\hline aug-cc-pV[Q5]Z-PP & & 2.2146 & 268.6 & 1.02 \\
\hline
\end{tabular}

frequencies. However, for these properties the use of different geminal exponents seems to be less important. It appears that the largest part of the improvement comes from the valence shell. One may note that both for equilibrium distances and for harmonic frequencies the VDZ-F12 results for $\mathrm{Br}_{2}, \mathrm{As}_{2}$ and $\mathrm{Ga}_{2}$ seem slightly better than the VTZ-F12 ones. This has also been observed by Peterson and Hill[39] and is probably due to some fortuitous error cancellation due to intramolecular BSSE effects. If the much larger aug-cc-pwCVnZ-PP basis sets are used, a smooth convergence pattern is found.

A drawback of the calculations shown so far is the dependence of the optimum geminal exponents on the atom or molecule. In calculations for molecules containing different heavy atoms one will have to use compromise values. In Tables 9 and 10 we compare results for the dimers and some halides obtained the same set of three exponents for all molecules. These were chosen to be $(0.8,1.5,1.9)$, $(0.9,1.6,2.2)$, and $(1.0,1.7,2.3)$ for double- $\zeta$, triple- $\zeta$, and quadruple- $\zeta$, respectively. Using these values the equilibrium distances and harmonic wave numbers change at most by $0.001 \mathrm{~A}$ and $1.2 \mathrm{~cm}^{-1}$, respectively, even for the double- $\zeta$ basis sets. 
Table 10. Computed spectroscopic constants of gallium and copper halides using different $\operatorname{CCSD}(\mathrm{T})-\mathrm{F} 12 \mathrm{x}$ approximations with 3 geminals (see text). $\omega_{e}$ and $\omega_{e} x_{e}$ in $\mathrm{cm}^{-1}$.

\begin{tabular}{|c|c|c|c|c|}
\hline Basis & Method & $R_{e} / \mathrm{A}$ & $\omega_{e}$ & $\omega_{e} x_{e}$ \\
\hline \multicolumn{5}{|l|}{ GaCl: } \\
\hline cc-pVDZ-F12 & $\mathrm{F} 12 \mathrm{a}$ & 2.2017 & 366.9 & 1.26 \\
\hline cc-pVTZ-F12 & $\mathrm{F} 12 \mathrm{a}$ & 2.2041 & 364.6 & 1.25 \\
\hline cc-pVQZ-F12 & F12a & 2.2026 & 365.7 & 1.25 \\
\hline cc-pVDZ-F12 & $\mathrm{F} 12 \mathrm{~b}$ & 2.2008 & 367.8 & 1.27 \\
\hline cc-pVTZ-F12 & $\mathrm{F} 12 \mathrm{~b}$ & 2.2038 & 364.9 & 1.25 \\
\hline cc-pVQZ-F12 & $\mathrm{F} 12 \mathrm{~b}$ & 2.2026 & 365.8 & 1.25 \\
\hline cc-pVDZ-F12 & $\left(\mathrm{F} 12^{*}\right)$ & 2.2025 & 367.3 & 1.27 \\
\hline cc-pVTZ-F12 & $\left(\mathrm{F} 12^{*}\right)$ & 2.2042 & 365.3 & 1.25 \\
\hline cc-pVQZ-F12 & $\left(\mathrm{F} 12^{*}\right)$ & 2.2024 & 366.1 & 1.25 \\
\hline aug-wCV[Q5]Z-PP & & 2.2000 & 367.4 & 1.27 \\
\hline Experiment & & 2.2017 & 365.3 & 1.2 \\
\hline \multicolumn{5}{|l|}{$\mathrm{CuCl}^{a}$ : } \\
\hline cc-pVDZ-F12 & $\mathrm{F} 12 \mathrm{a}$ & 2.0521 & 415.8 & 1.60 \\
\hline cc-pVTZ-F12 & $\mathrm{F} 12 \mathrm{a}$ & 2.0486 & 417.7 & 1.61 \\
\hline cc-pVQZ-F12 & $\mathrm{F} 12 \mathrm{a}$ & 2.0470 & 418.2 & 1.62 \\
\hline cc-pVDZ-F12 & $\mathrm{F} 12 \mathrm{~b}$ & 2.0531 & 414.4 & 1.58 \\
\hline cc-pVTZ-F12 & $\mathrm{F} 12 \mathrm{~b}$ & 2.0489 & 417.2 & 1.61 \\
\hline cc-pVQZ-F12 & $\mathrm{F} 12 \mathrm{~b}$ & 2.0470 & 418.0 & 1.61 \\
\hline cc-pVDZ-F12 & $\left(\mathrm{F} 12^{*}\right)$ & 2.0559 & 413.0 & 1.56 \\
\hline cc-pVTZ-F12 & $\left(\mathrm{F} 12^{*}\right)$ & 2.0497 & 416.8 & 1.59 \\
\hline cc-pVQZ-F12 & $\left(\mathrm{F} 12^{*}\right)$ & 2.0471 & 418.0 & 1.61 \\
\hline aug-wCV[Q5]Z-PP & & 2.0471 & 418.8 & 1.59 \\
\hline Experiment & & 2.0512 & 415.3 & 1.58 \\
\hline \multicolumn{5}{|l|}{$\mathrm{CuBr}^{a}:$} \\
\hline cc-pVDZ-F12 & $\mathrm{F} 12 \mathrm{a}$ & 2.1716 & 313.8 & 0.93 \\
\hline cc-pVTZ-F12 & $\mathrm{F} 12 \mathrm{a}$ & 2.1684 & 315.8 & 0.96 \\
\hline cc-pVQZ-F12 & $\mathrm{F} 12 \mathrm{a}$ & 2.1669 & 316.0 & 0.94 \\
\hline cc-pVDZ-F12 & $\mathrm{F} 12 \mathrm{~b}$ & 2.1732 & 312.4 & 0.92 \\
\hline cc-pVTZ-F12 & F12b & 2.1688 & 315.2 & 0.95 \\
\hline cc-pVQZ-F12 & $\mathrm{F} 12 \mathrm{~b}$ & 2.1669 & 315.8 & 0.94 \\
\hline cc-pVDZ-F12 & $\left(\mathrm{F} 12^{*}\right)$ & 2.1757 & 311.7 & 0.91 \\
\hline cc-pVTZ-F12 & $\left(\mathrm{F} 12^{*}\right)$ & 2.1696 & 315.2 & 0.94 \\
\hline cc-pVQZ-F12 & $\left(\mathrm{F} 12^{*}\right)$ & 2.1670 & 315.9 & 0.94 \\
\hline aug-cc-p[Q5]Z-PP & & 2.1663 & 316.4 & 0.94 \\
\hline Experiment & & 2.1734 & 314.8 & 0.96 \\
\hline
\end{tabular}

In most cases the changes are even smaller. An exception is $\mathrm{Cu}_{2}$ with the aug-ccpVDZ-PP basis set; in this case the distance shortens by $0.003 \mathrm{~A}$, but the values in Table 9 with the compromise exponents are more accurate than those in Table 8. As already noted earlier, the larger exponents yield better results for $\mathrm{Cu}_{2}$.

In Tables 9 and 10 we also compare the $\operatorname{CCSD}(\mathrm{T})-\mathrm{F} 12 \mathrm{a},-\mathrm{F} 12 \mathrm{~b}$, and $\left(\mathrm{F} 12^{*}\right)$ approximations. Since currently we have only a closed-shell implementation of $\operatorname{CCSD}\left(\mathrm{F} 12^{*}\right)$, there are no dissociation energies and open-shell molecules in this comparison. The F12a and F12b approximations yield very similar results in all cases. The differences to $\left(\mathrm{F} 12^{*}\right)$ are somewhat larger; naturally, they decrease with increasing basis set. No clear conclusion is possible about which is the most accurate method; for $\mathrm{Br}_{2}, \mathrm{As}_{2}$ the $\left(\mathrm{F} 12^{*}\right)$ results for the $\mathrm{DZ}$ and $\mathrm{TZ}$ basis sets look slightly better, but the opposite appears to be the case for $\mathrm{Cu}_{2}$ and the halides. We also found in other cases that the relative accuracy of the various 
F12 approximations depends on the molecule and considered property, but the differences between these methods are usually smaller than the intrinsic error of the $\operatorname{CCSD}(\mathrm{T})$ method at its CBS limit. One may therefore safely use the F12b approximation, which is computationally significantly cheaper than $\left(\mathrm{F} 12^{*}\right)$.

In the Tables we also reported results obtained by extrapolating the correlation energies obtained with the large aug-cc-pwCVQZ and aug-cc-pwCV5Z basis sets (aug-cc-pVQZ-PP and aug-cc-pV5Z-PP in case of $\mathrm{Cu}_{2}$ ) using $E_{n}=E_{\mathrm{CBS}}+A / n^{3}$, where $n$ is the cardinal number of the basis set. The best F12 results are quite close to the extrapolated values, but it is not clear which results are more accurate. According to previous experience, it may well be that the quadruple- $\zeta$ F12 results are closer to the true basis set limits than the extrapolated ones. It should be noted, however, that the triples contributions are not explicitly correlated, and therefore introduce some remaining basis set truncation errors.

A final note concerns the comparison of the $\operatorname{CCSD}(\mathrm{T})-\mathrm{F} 12 \mathrm{~b}$ values with the experimental values. Despite the fact that the VQZ-F12 results should be very close to the basis set limits, the deviations from the experimental values are quite large. This is due to surprisingly large contributions of higher excitations; for example, the CCSDTQ - CCSD $(\mathrm{T})$ correction for the equilibrium distances of $\mathrm{Br}_{2}$ and $\mathrm{As}_{2}$ amounts to about $0.0024 \mathrm{~A}$ and $0.0043 \mathrm{~A}$, respectively, and even this does not cover the full contribution of higher excitations. The effect comes mainly from the valence electrons, and is probably due to the rather small HOMO-LUMO gap in these molecules. In contrast to $\operatorname{CCSD}(\mathrm{T})$, internally contracted MRCI calculations[54] with full valence CASSCF reference functions yields excellent agreement with experimental values, if the $c c+c v$ corrections are added. A more detailed investigation of these effects will be published elsewhere[52].

\section{Conclusions}

A modification of the MP2-F12 and CCSD-F12x methods has been proposed and tested, in which different geminal exponents are used for the correlation of corecore, core-valence and pure valence orbital pairs. It has been demonstrated for a number of diatomic molecules involving $d$-shell correlation that this leads to much improved correlation energies and molecular properties. Most important is an additional exponent for core-valence correlation, while the pure core-core correlation has - despite its huge contribution to the correlation energy - hardly any effect on the equilibrium distances and vibrational frequencies. The additional computational cost for using two or three exponents is rather small, since only terms that scale with $\mathcal{O}\left(N^{3}\right)$ and $\mathcal{O}\left(N^{4}\right)$ are affected. The dominating $\mathcal{O}\left(N^{5}\right)$ terms in MP2-F12 and $\mathcal{O}\left(N^{6}\right)$ ones in CCSD-F12 are entirely unchanged.

The results demonstrate that F12 methods are extremely well suited to treat inner-shell correlation effects and eliminate the frustratingly slow convergence of core-valence effects with basis set size. This makes it possible to use much smaller orbital basis sets than in standard $\operatorname{CCSD}(\mathrm{T})$ calculations. The new cc-pVnZ-F12PP basis sets of Peterson and Hill, which have been used here for $\mathrm{Br}$, As, and $\mathrm{Ga}$, are only marginally larger than pure valence basis sets; they just include one additional steep $d$ and one $f$ function. Our results show that with appropriately chosen geminal exponents near basis set limit results are obtained at the triple- $\zeta$ level. Even with just double- $\zeta$ basis sets very good accuracy is achieved. Thus, it can be expected that the current method will be very useful, e.g. for transition metal complexes, as long as there is a clear distinction between core and valence orbitals. Further systematic studies for transition metal compounds are under way. 


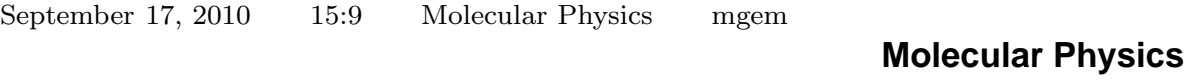

\section{Acknowledgments}

This work was funded by the DFG as part of a NSF-DFG grant and by the Stuttgart SimTech Cluster of Excellence. HJW also acknowledges generous support from the Fonds der Chemischen Industrie. We are grateful to Kirk Peterson and Grant Hill for providing their new basis sets prior to publication and for many helpful discussions. HJW thanks C. Hättig and D. Tew for providing test results for their $\left(\mathrm{F} 12^{*}\right)$ method, which were very useful for debugging our implementation.

\section{References}

[1]W. Klopper, F.R. Manby, S. Ten-no and E.F. Valeev, Int. Rev. Phys. Chem. 25, 427 (2006).

[2]D. Tew, C. Hättig, R. Bachorz and W. Kopper, in Recent Progress in Coupled Cluster Methods, ed. P. Ćársky, J. Paldus, J. Pittner, pp. 535-572 (Spinger, 2010).

[3]H.-J. Werner, T.B. Adler, G. Knizia and F.R. Manby, in Recent Progress in Coupled Cluster Methods, ed. P. Čársky, J. Paldus, J. Pittner, pp. 573-619 (Spinger, 2010).

[4]T. Kato, Comm. Pure Appl. Math. 10, 151-177 (1957).

[5]R.T. Pack and W. Byers Brown, J. Chem. Phys. 45, 556-559 (1966).

[6]S. Ten-no, J. Chem. Phys. 121, 117-129 (2004).

[7]S. Ten-no, Chem. Phys. Lett. 398, 56-61 (2004).

[8] G. Knizia, T.B. Adler and H.-J. Werner, J. Chem. Phys. 130, 054104 (2009).

[9] G. Rauhut, G. Knizia and H.-J. Werner, J. Chem. Phys. 130, 054105 (2009)

[10] O. Marchetti and H.-J. Werner, J. Phys. Chem. A 113, 11580-11585 (2009).

[11]T.B. Adler, H.-J. Werner and F.R. Manby, J. Chem. Phys. 130, 054106 (2009).

[12]E.F. Valeev, J. Chem. Phys. 125, 244106 (2006).

[13] T. Shiozaki, M. Kamiya, S. Hirata and E.F. Valeev, Phys. Chem. Chem. Phys. 10, 3358 (2008).

[14]A. Köhn, G.W. Richings and D.P. Tew, J Chem Phys 129, 201103 (2008).

[15]T. Shiozaki, M. Kamiya, S. Hirata and E.F. Valeev, J. Chem. Phys. 130, 054101 (2009).

[16]A. Köhn, J. Chem. Phys. 130, 131101 (2009).

[17]H. Fliegl, W. Klopper and C. Hättig, J. Chem. Phys. 122, 084107 (2005).

[18] H. Fliegl, C. Hättig and W. Klopper, Int. J. Quantum Chem. 106, 2306 (2006).

[19] D.P. Tew, W. Klopper, C. Neiss and C. Hättig, Phys. Chem. Chem. Phys. 9, 1921-1930 (2007).

[20] D.P. Tew, W. Klopper and C. Hättig, Chem. Phys. Lett. 452, 326-332 (2008).

[21]T.B. Adler, G. Knizia and H.-J. Werner, J. Chem. Phys. 127, 221106 (2007).

[22] M. Torheyden and E.F. Valeev, Phys. Chem. Chem. Phys. 10, 3410-3420 (2008).

[23] E.F. Valeev and T.D. Crawford, J. Chem. Phys. 128, 244113 (2008).

[24]T. Shiozaki, E.F. Valeev and S. Hirata, J. Chem. Phys. 131, 044118 (2009).

[25] C. Hättig, D.P. Tew and A. Köhn, J. Chem. Phys. 132, 231102 (2010).

26] H.-J. Werner, T.B. Adler and F.R. Manby, J. Chem. Phys. 126, 164102 (2007).

[27] G. Knizia and H.-J. Werner, J. Chem. Phys. 128, 154103 (2008).

[28] W. Klopper, Chem. Phys. Lett. 186, 583-585 (1991).

[29]E.F. Valeev, Chem. Phys. Lett. 395, 190-195 (2004).

[30]H.-J. Werner, P.J. Knowles, R. Lindh, F.R. Manby and M. Schütz et al., MOLPRO, version 2009.1, a package of ab initio programs Cardiff, UK, 2009, see http://www.molpro.net.

[31]F.R. Manby, J. Chem. Phys. 119, 4607-4613 (2003).

[32] A.J. May and F.R. Manby, J. Chem. Phys. 121, 4479-4485 (2004).

[33]S. Obara and A. Saika, J. Chem. Phys. 84 (7), 3963-3974 (1986).

[34] S. Obara and A. Saika, J. Chem. Phys. 89 (3), 1540-1559 (1988).

[35]R. Ahlrichs, Phys. Chem. Chem. Phys. 8, 3072-3077 (2006).

[36]R. Ahlrichs, Phys. Chem. Chem. Phys. 6, 5119-5121 (2004).

[37]S. Höefener, F.A. Bischoff, A. Glöß and W. Klopper, Phys. Chem. Chem. Phys. 10, 3390-3399 (2008).

[38]F.R. Manby, H.-J. Werner, T.B. Adler and A.J. May, J. Chem. Phys. 124, 094103 (2006).

[39] K.A. Peterson and J.G. Hill (2010), private communication.

[40] J.G. Hill, S. Mazumder and K.A. Peterson, J. Chem. Phys. 132, 054108 (2010).

[41] K.A. Peterson and C. Puzzarini, Theor. Chem. Acc. 114, 283-296 (2005).

[42] J.G. Hill (2010), private communication.

[43] A. Hellweg, C. Haettig, S. Hoefener and W. Klopper, Theor. Chem. Acc. 117, 587-597 (2007).

[44] T.H. Dunning, Jr., K.A. Peterson and A.K. Wilson, J. Chem. Phys. 114, 9244-9253 (2001).

[45] K.E. Yousaf and K.A. Peterson, Chem. Phys. Lett. 476, 303-307 (2009).

[46]F. Weigend, J. Comput. Chem. 29, 167-175 (2007).

[47]K.A. Peterson, D. Figgen, E. Goll, H. Stoll and M. Dolg, J. Chem. Phys. 119, 11113 (2003).

[48] B. Metz, H. Stoll and M. Dolg, J. Chem. Phys. 113, 2563 (2000).

[49]D. Figgen, G. Rauhut, M. Dolg and H. Stoll, Chem. Phys. 311, 227 (2005).

[50]F.A. Bischoff, S. Höfener, A. Glöss and W.Klopper, Theor. Chem. Acc. 121, 11-19 (2008).

[51]P.J. Knowles, C. Hampel and H.-J. Werner, J. Chem. Phys. 99 (7), 5219-5227 (1993).

[52] C. Krause, H. Stoll, K.A. Peterson and H.-J. Werner (2010), to be published.

[53] K. Peterson and K. Yousaf (2010), private communication.

[54] H.-J. Werner and P.J. Knowles, J. Chem. Phys. 89, 5803-5814 (1988). 


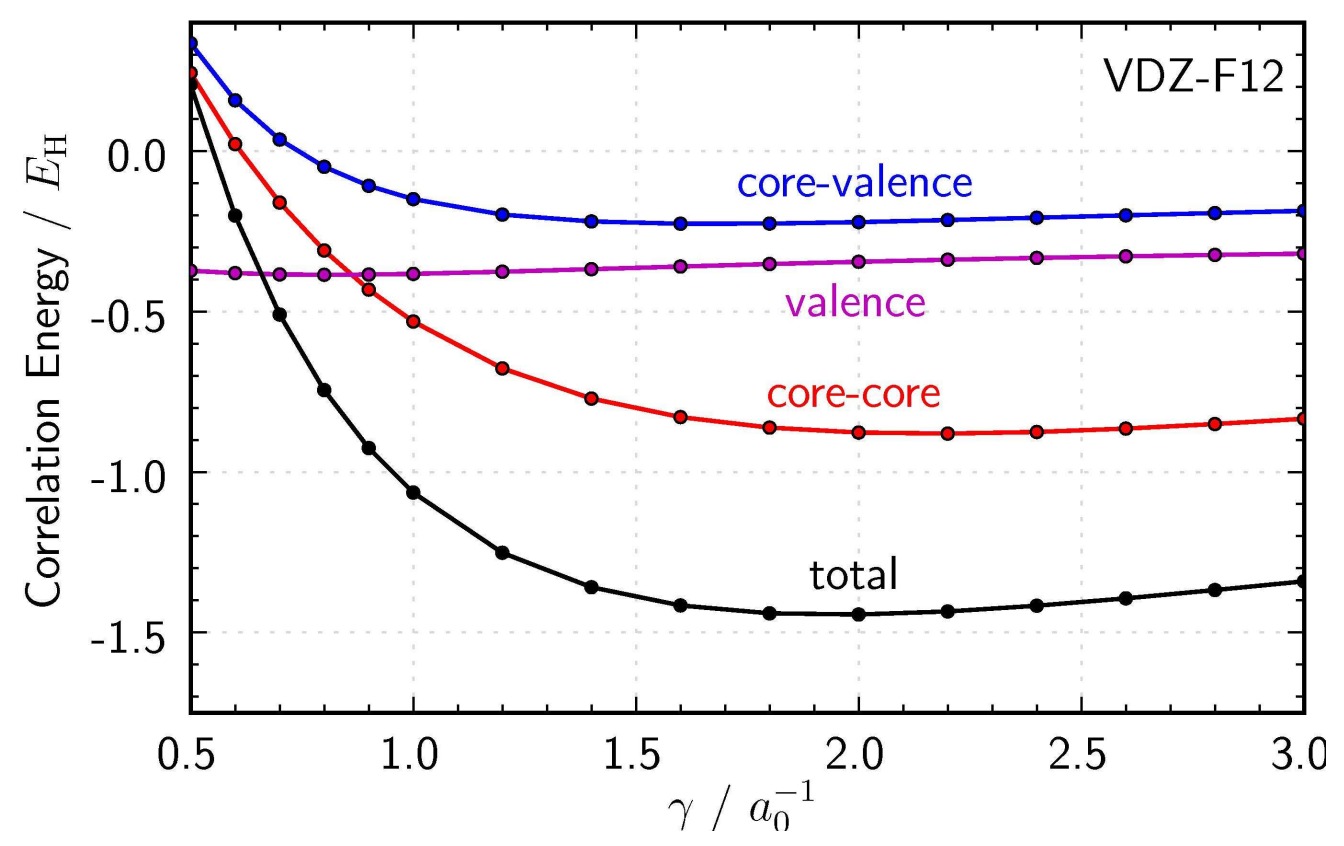

$115 \times 71 \mathrm{~mm}(600 \times 600 \mathrm{DPI})$ 


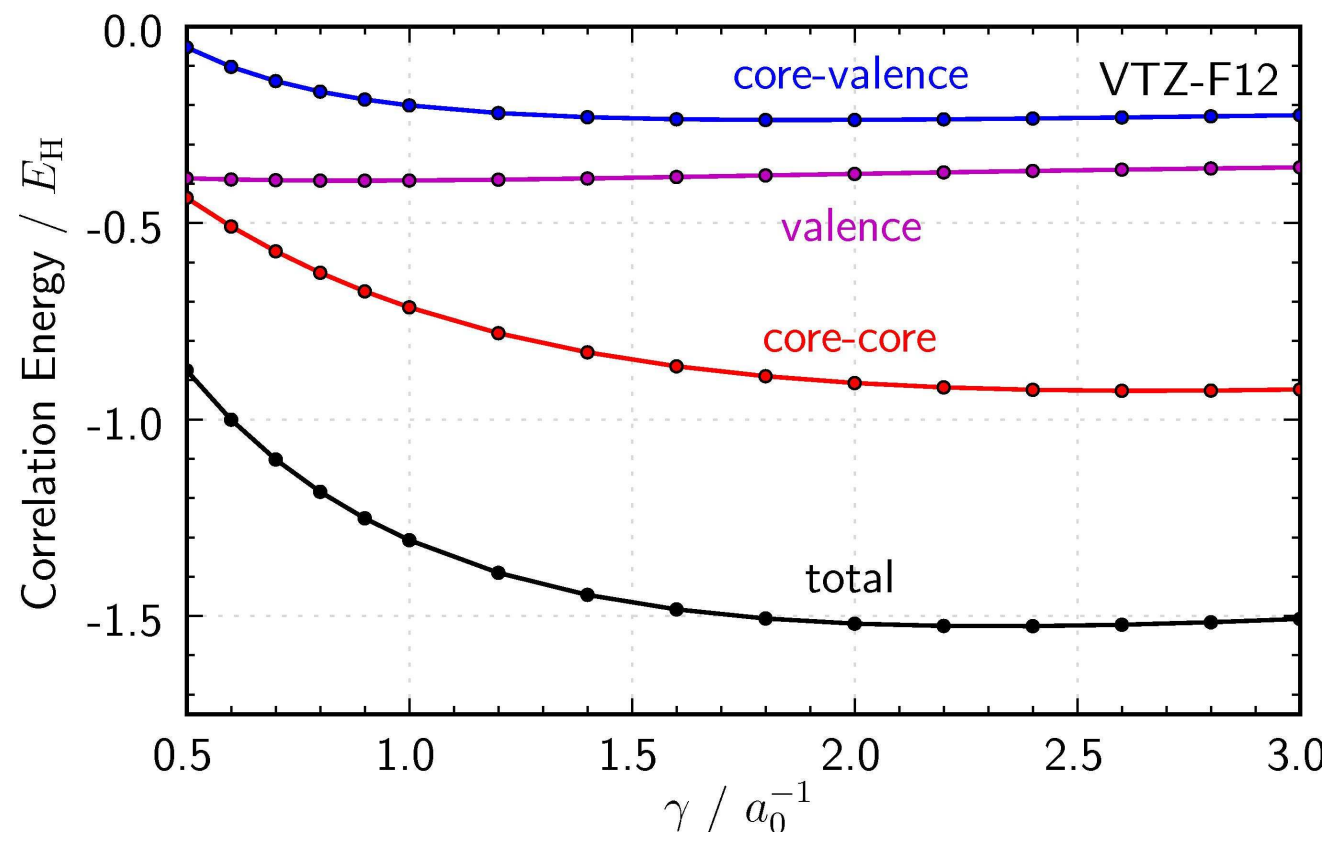

$115 \times 72 \mathrm{~mm}(600 \times 600 \mathrm{DPI})$ 


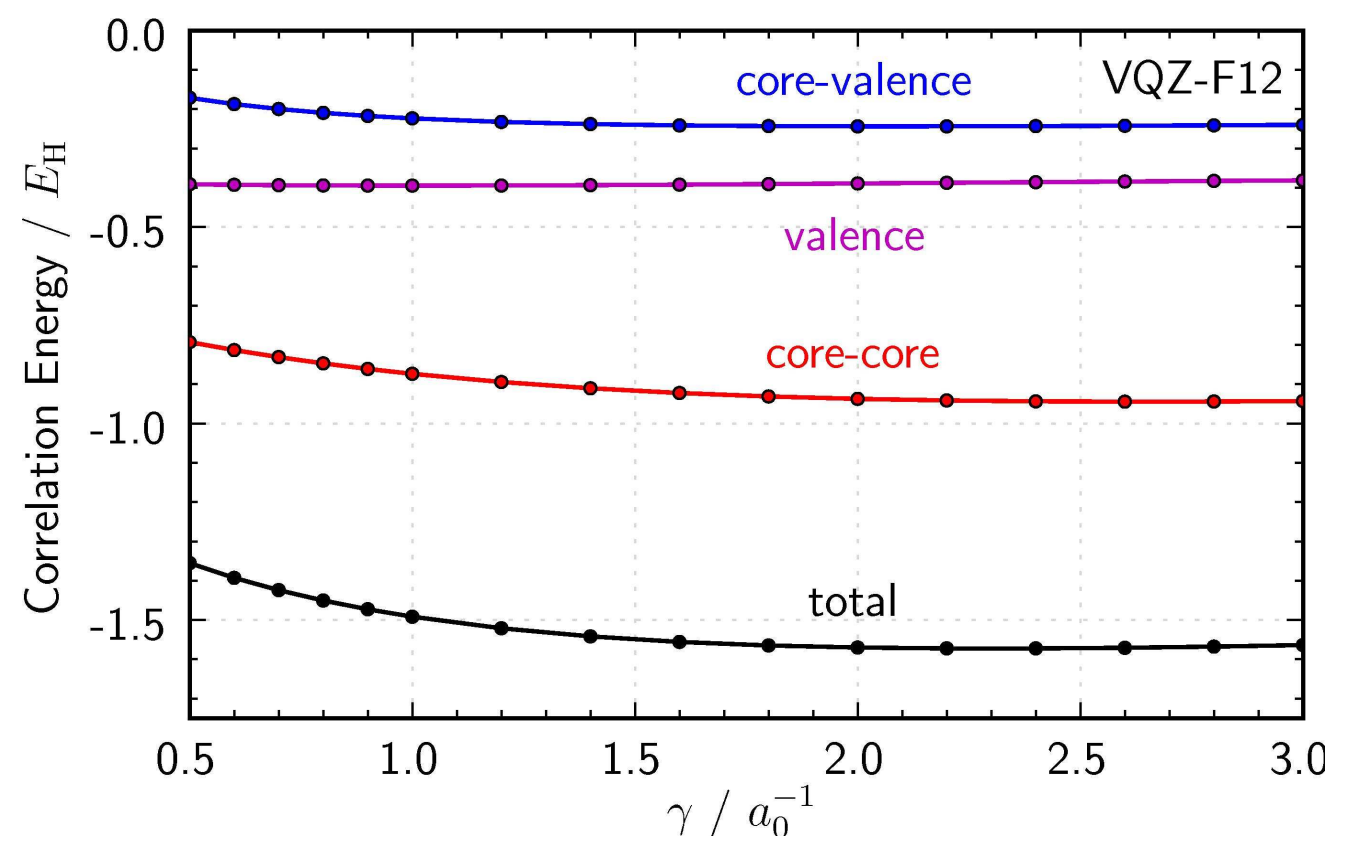

$115 \times 72 \mathrm{~mm}(600 \times 600 \mathrm{DPI})$ 


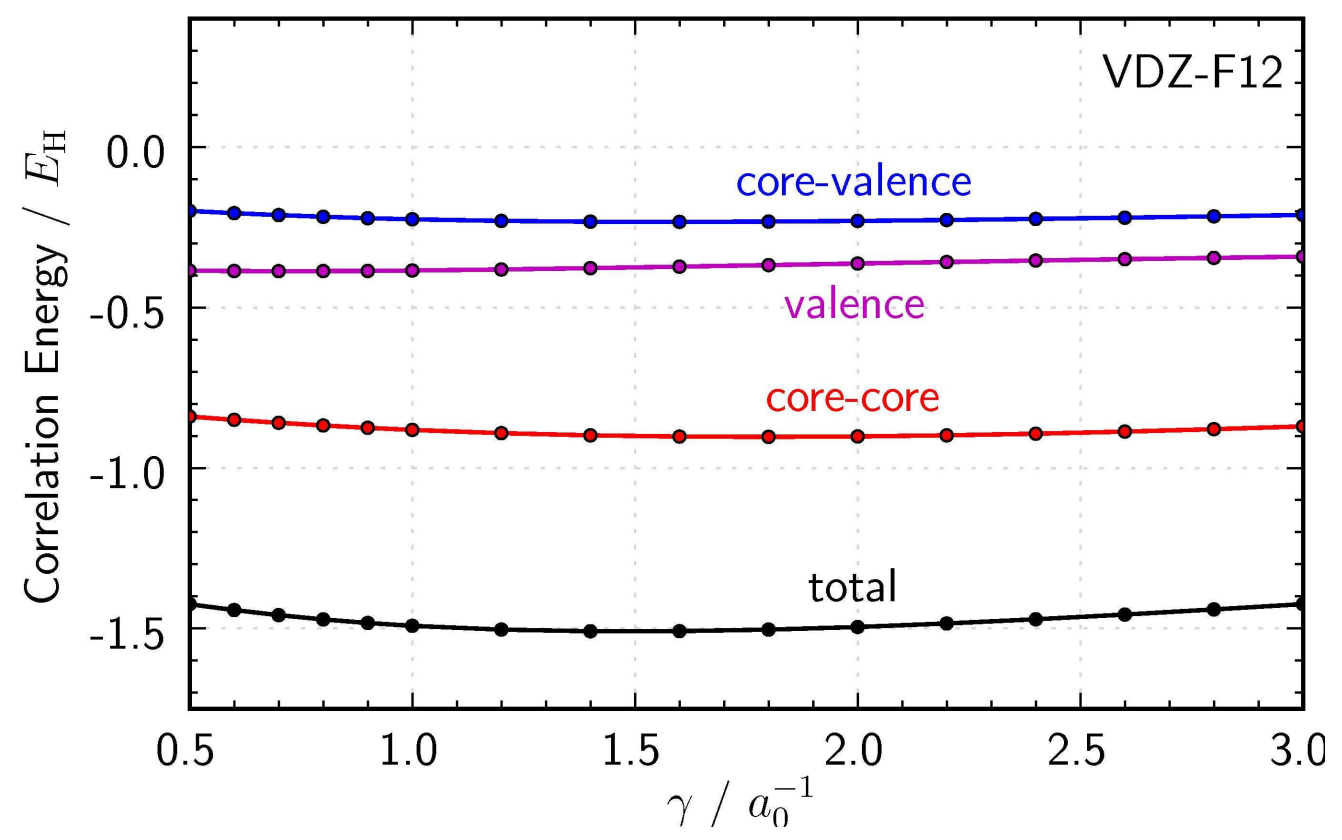

$115 \times 71 \mathrm{~mm}(600 \times 600$ DPI $)$ 


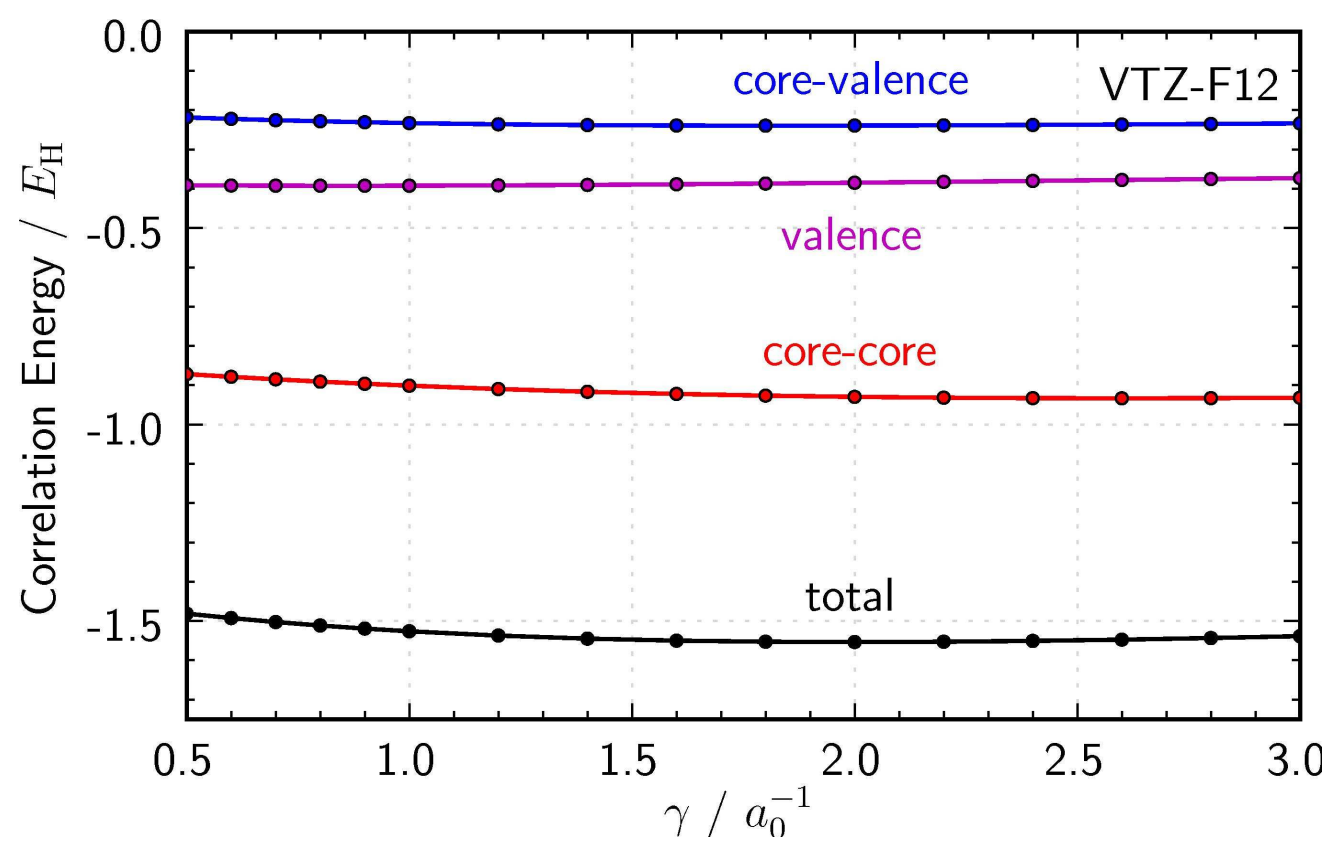

$115 \times 72 \mathrm{~mm}(600 \times 600 \mathrm{DPI})$ 


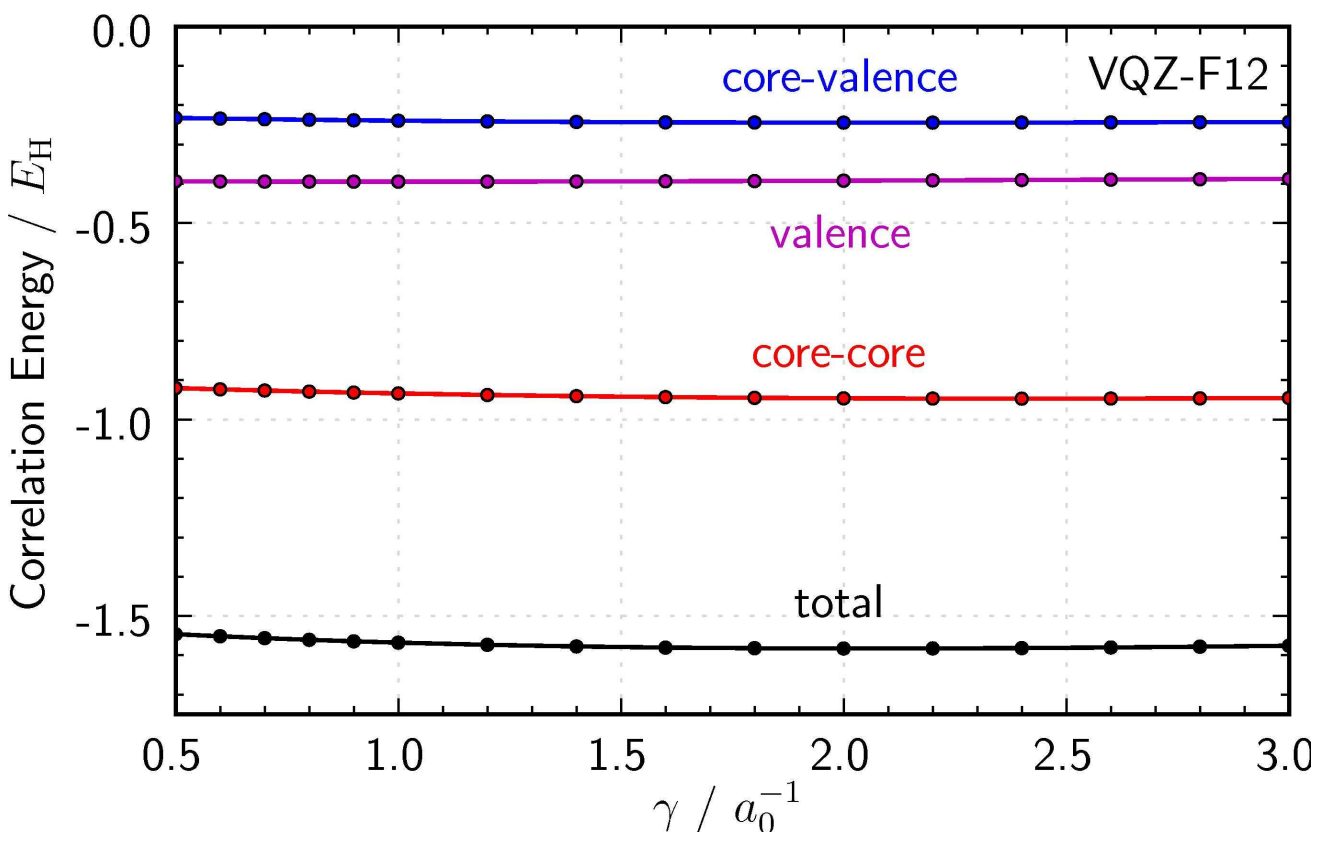

$115 \times 72 \mathrm{~mm}(600 \times 600 \mathrm{DPI})$ 